\title{
Analysis and Design of Biological Circuits and Systems
}

\author{
Heinz Koeppl \\ School of Communication and Computer Sciences \\ Ecole Polytechnique Federale de Lausanne (EPFL) \\ Station 14, 1015 Lausanne, Switzerland \\ Email: heinz.koeppl@epfl.ch
}

\author{
Gianluca Setti \\ Department of Engineering (ENDIF) \\ University of Ferrara \\ Via Saragat, 1, 44100 Ferrara, Italy \\ Email: gianluca.setti@unife.it
}

\begin{abstract}
Systems and synthetic biology are two emerging disciplines that hold promise to revolutionize our understanding of biological systems and to herald a new era of programmable hardware, respectively. Mathematical abstraction and today's abundance of quantitative biological data enables the up-scaling of analysis and design methodologies. In this tutorial paper we provide an engineering-centered introduction to those disciplines. Biological key concepts such as the central dogma of molecular biology are discussed, descriptions of bio-molecular reaction networks in terms of continuous-time Markov processes and ordinary differential equations are reviewed. Topological analysis of networks is introduced and the methods of metabolic flux balance analysis and elementary flux modes or extreme pathways are discussed.
\end{abstract}

\section{INTRODUCTION}

With the advent of high-throughput experiments, such as DNA micro-array, chromatin immunoprecipitation-on-chip (ChIP-chip) and fluorescence-activated cell sorting (FACS) [1] the molecular biologist is confronted with an abundance of detailed biochemical information for the analysis of his particular system under study. On the other side the upscaling of technologies such as oligonucleotide synthesis today allows the cheap production of custom DNA sequences [2] that form the basis for the design of new artificial circuits or the replication of subsystems of natural circuits. To challenge the problems in the analysis and the design of biological systems effort has to be put on the process of formalization and standardization analogous to that electronics was undergoing in the last forty years. The founding of systems and synthetic biology initiated this effort [3].

The analysis of biological circuits from experimental observations in most cases does not permit the determination of a simple underlying mechanism that could explain the data. In general, the more data is available the more the intricates of the mechanism are uncovered. As a consequence the explanatory mechanism can hardly be found by intuitive reasoning even though biologist are extremely skilled in their calculus of successive and concurrent inhibition and activation. Biochemists and molecular biologists already developed a large set of methods that facilitates a quantitative analysis of cellular processes [4]-[6].

For the redesign of biological systems current efforts in synthetic biology goes beyond recombinant DNA technologies of bioengineering and considers the bottom-up design of circuits by utilizing the fast evolving technology to synthesize long
DNA polymers base-pair per base-pair [2]. The technology enables the design of small canonical parts such as inverters, amplifiers and measurement circuitries [3].

The paper proceeds as follows. In Section II we introduce the basic concepts of molecular biology. The main part of the paper is Section III where we outline the different modeling approaches for biochemical reaction systems that serve as the basis for the analysis and design of biological circuits and systems. In Section IV we review the topological analysis that is particularly suited for large metabolic networks and finally in Section $\mathrm{V}$ we draw conclusions.

\section{THE CENTRAL DOGMA OF MOLECULAR BIOLOGY}

The central dogma of molecular biology was formulated by F. Crick in 1958 and describe the flow of sequence information between the main biopolymers DNA, RNA and proteins with in the cell [1] (cf. Fig. 1). The dogma states that among the six potential information flows between these biopolymers only two are predominant. That are the flow from DNA to RNA (transcription) and from RNA to protein (translation). Although considered today as an oversimplification the dogma forms the basis to disentangle the diverse interactions happening within a cell. A complicated recurrent network results if one takes into account the interaction among proteins and the action of transcription factor proteins on DNA.

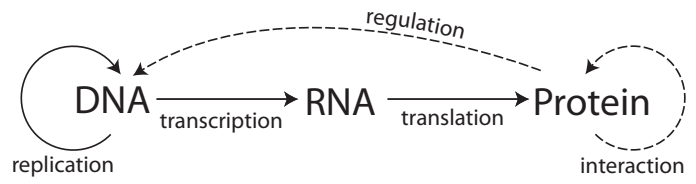

Fig. 1. The central dogma of molecular biology; the transfer of sequence information follows the linear chain from DNA to RNA to proteins; dashed lines do not transfer sequence information but indicate possible interactions.

\section{MATHEMATICAL ABSTRACTION}

The cell is a complex molecular machine that contains a large number of structural elements such as membranes, scaffolds, compartments, filaments and even cellular motors as the flagella motor in $E$. coli [1]. An important concept for the formalization of cellular processes that we are going to focus on in the following is that of a chemical reaction. Consider a set of molecular species or agents $\mathcal{A} \equiv\left\{A_{1}, A_{2}, \ldots, A_{N}\right\}$ 
with $N \in \mathbb{N}$ and a set of reactions $\mathcal{R} \equiv\left\{R_{1}, R_{2}, \ldots, R_{M}\right\}$, $M \in \mathbb{N}$. A reaction $R_{k}$ among different species is defined by the construct

$$
\begin{aligned}
R_{k}: \quad S_{1 k} A_{1}+S_{2 k} A_{2}+\cdots+S_{N k} A_{N} \\
\quad \stackrel{c_{k}}{\longrightarrow} P_{1 k} A_{1}+P_{2 k} A_{2}+\cdots+P_{N k} A_{N}
\end{aligned}
$$

where here the symbol "+" indicates the chemical composition of species and not the algebraic addition. The likelihood for the reaction to occur is given by reaction rate constant $c_{k} \in \mathbb{R}_{0}$. We denote $S_{i k} \in \mathbb{N}_{0}$ and $P_{i k} \in \mathbb{N}_{0}$ as the stoichiometric coefficients for the substrates (left-hand-side) and the products (right-hand-side) of reaction $R_{k}$, respectively. Moreover we denote the matrix $\mathbf{N} \in \mathbb{Z}^{N \times M}$ as the stoichiometric matrix of the tuple $(\mathcal{A}, \mathcal{R})$ the elements of which are defined as $N_{i k} \equiv$ $P_{i k}-S_{i k}$. Thus for a given reaction $R_{k}$ the $k$-th column of $\mathbf{N}$ corresponds to the net consumption or production of the involved molecular species.

Reactions take place in physical time and space. According to the level of detail of the chosen description one may neglect the physical space by assuming that the ensemble of molecular agents are fast mixing and that the propensity of a reaction to happen is independent of the actual position of the agents that participate in that reaction. In physical terms this assumption is feasible if the time-scale of diffusion of agents within the volume is much faster than that of any reaction in $\mathcal{R}$. Macromolecular crowding [7], i.e., the occupancy of 20$30 \%$ of the total cell volume with macromolecules as well as other experimental findings such as membrane recruitment processes indicate the limits of the fast diffusion assumption. Quantitative attempts to account for spatial phenomena have been made through reaction-diffusion partial differential equations and compartmental models [8] as well as through particle tracking methods based on the Smoluchowski diffusion model [9].

\section{A. Chemical master equation}

In the following we describe the stochastic kinetic theory of coupled chemical reactions under assumption of fast mixing or well-stirredness. We then need to associate with every molecular species $A_{k}$ only a scalar random variable $X_{k}(t)$ denoting the number of the copies of $A_{k}$ in the ensemble at time $t$. The collection $\mathbf{X}(t) \equiv\left(X_{1}(t), \ldots, X_{N}(t)\right)^{T}$ taking values in the reachable set $\mathcal{X} \subseteq \mathbb{N}_{0}^{N}$ fully determines the state of the reaction system at time $t$. In the following, we will refer to a realization of a random variable by its lower-case version. Based on thermodynamic principles the evolution of $\mathbf{X}(t)$ was shown [10] to follow a continuous-time Markov jump process (CTMJP). The process defines a probability distribution $p: \mathcal{X} \times \mathbb{R}_{0} \mapsto[0,1]$ over state $\mathbf{x} \in \mathcal{X}$ and time

$$
p(\mathbf{x}, t) \equiv P\left(\mathbf{X}(t)=\mathbf{x} \mid \mathbf{X}(0)=\mathbf{x}_{0}\right) .
$$

The probability that in the time interval $(t, t+\mathrm{d} t]$ a single $R_{k}$ occurs from a state $\mathrm{x}$ is

$$
P\left(\mathbf{X}(t+\mathrm{d} t)=\mathbf{x}+\mathbf{N e}_{k} \mid \mathbf{X}(t)=\mathbf{x}\right)=a_{k}(\mathbf{x}) \mathrm{d} t
$$

with $\mathbf{e}_{k}$ the $k$-th basis vector of an $M$-dimensional basis. We refer to $a_{k}(\mathbf{x})$ as the propensity or hazard function of $R_{k}$ at $\mathrm{x}$. For mass-action kinetics hazard functions take the form

$$
a_{k}(\mathbf{x}) \equiv c_{k} \prod_{j=1}^{N}\left(\begin{array}{c}
x_{j} \\
S_{j k}
\end{array}\right),
$$

containing the binomial coefficient for the various combinations reaction $R_{k}$ can take place. By conservation of probability the increment or decrement of the probability $p(\mathbf{X}, t)$ for the state $\mathbf{X}$ at time $t$ can happen through firing of reactions from state $\mathbf{X}$ and firing of reactions that cause the system to reach state $\mathbf{X}$, respectively. For the infinitesimal case these firings are assumed to give independent ways to change $p(\mathbf{X}, t)$. Thus we obtain the additive relation

$$
\begin{aligned}
& \frac{\partial p(\mathbf{x}, t)}{\partial t}= \\
& \sum_{j=1}^{M} a_{j}\left(\mathbf{x}-\mathbf{N e}_{j}\right) p\left(\mathbf{x}-\mathbf{N e}_{j}, t\right)-\sum_{j=1}^{M} a_{j}(\mathbf{x}) p(\mathbf{x}, t),
\end{aligned}
$$

which is known as the chemical master equation and is equivalent to the Chapman-Kolmogorov forward equation of Markov processes [11] for the particular case of chemical kinetics. Consulting (1) reveals that the corresponding transition kernel has no explicit time-dependence and we are thus concerned with a time-invariant Markov process. Due to the exponential scaling of discrete states with $N$ the computational complexity to numerically solve (3) becomes prohibitively large for systems of moderate size.

\section{B. Rate equation}

Rate equations are the most common way to describe the dynamics of coupled chemical reactions. They can be considered to give an approximation to the dynamics of $\mathrm{E}[\mathbf{X}(t)]$, where $\mathrm{E}[\cdot]$ denotes the expectation with respect to $p(\mathbf{X}, t)$. Taking into (3) we develop the exact expression for the temporal increment of the mean value as

$$
\begin{aligned}
\frac{\mathrm{d}}{\mathrm{d} t} & \mathrm{E}[\mathbf{X}(t)]=\sum_{\mathbf{x} \in \mathcal{X}} \mathbf{x} \frac{\partial p(\mathbf{x}, t)}{\partial t} \\
& =\sum_{j=1}^{M}\left\{\mathrm{E}\left[\left(\tilde{\mathbf{X}}(t)+\mathbf{N e}_{j}\right) a_{j}(\tilde{\mathbf{X}}(t))\right]-\mathrm{E}\left[\mathbf{X}(t) a_{j}(\mathbf{X}(t))\right]\right\} \\
& =\sum_{j=1}^{M} \mathrm{E}\left[\mathbf{N e}_{j} a_{j}(\mathbf{X}(t))\right]
\end{aligned}
$$

where in line 2 we used (3) and applied the variable substitution $\tilde{\mathbf{X}} \equiv \mathbf{X}-\mathbf{N e}_{j}$. Finally we obtain

$$
\frac{\mathrm{d}}{\mathrm{d} t} \mathrm{E}[\mathbf{X}(t)]=\mathbf{N E}[\mathbf{a}(\mathbf{X}(t))],
$$

with the definition of the hazard vector $\mathbf{a}(\mathbf{X}) \equiv$ $\left(a_{1}(\mathbf{X}), \ldots, a_{M}(\mathbf{X})\right)^{T}$. For nonlinear hazard functions (2), the right-hand-side of (4) involves higher-order moments and thus (4) does not constitute a closed system of equations for the first-order moment. Applying the mean-field approximation $\mathrm{E}[\mathbf{a}(\mathbf{X}(t))] \approx \mathbf{a}(\mathrm{E}[\mathbf{X}(t)])$, justifiable for distributions $p(\mathbf{X}, t)$ that are unimodal and sharp for all $t \in \mathbb{R}_{0}$, we obtain the equation for the approximate mean $\hat{\mathbf{x}}(t) \approx \mathrm{E}[\mathbf{X}(t)]$ as

$$
\frac{\mathrm{d} \hat{\mathbf{x}}}{\mathrm{d} t}=\mathbf{N a}(\hat{\mathbf{x}}),
$$


that is now a closed system of autonomous ordinary differential equation for the approximate first-order moment and normally referred to as rate equation. Note that by the linearity of the expectation operator the mean field approximation is exact for reaction systems only containing zero-th and first order reaction, i.e., $R_{k}$ with $a_{k}(\mathbf{X})=c_{k}$ and $a_{k}(\mathbf{X})=$ $c_{k} X_{j}$ for some $1 \leq j \leq M$, respectively. The mean field approximation becomes better with increasing copy numbers for the species in $\mathcal{A}$ and $\hat{\mathbf{x}}(t)$ converges to $\mathrm{E}[\mathbf{X}(t)]$ for any $t \in \mathbb{R}_{0}$ in the limit of infinite copy numbers. Equivalent to this limit is the assumption of continuously divisible agents or species, i.e., $\mathcal{X} \subseteq \mathbb{R}_{0}^{N}$. Based on this continuity assumption a better approximation to (3) than the rate equation (5) can be achieved by a stochastic differential equation.

\section{Stochastic differential equation}

For sufficiently large copy numbers a CTMJP can well be approximated by a diffusion process described by a stochastic differential equation where naturally the state space is $\mathcal{X} \in$ $\mathbb{R}_{0}^{N}$. The corresponding approximate evolution equation for $p(\mathbf{X}, t)$ is a nonlinear Fokker-Planck equation [11]. For the approximation steps that lead to it, starting from (3) the reader is referred to [11]. An intuitive derivation of the stochastic differential equation is as follows. For a small time interval $(t, t+\Delta t]$ the propensities (2) can be assumed to be constant and the occurrence of reaction events follow a Poisson distribution $\operatorname{Po}(\cdot)$ independently for each $R_{k}$. Denote $\Delta Y_{k} \in \mathbb{N}_{0}$ as the number of $R_{k}$ reactions within $(t, t+\Delta t]$ then $\Delta Y_{k} \sim$ $\operatorname{Po}\left(a_{k}(\mathbf{X}) \Delta t\right)$. Recalling the definition for the mean and variance of a Poisson process we have $\mathrm{E}\left[\Delta Y_{k}\right]=a_{k}(\mathbf{X}) \Delta t$ and $\mathrm{E}\left[\Delta Y_{k} \Delta Y_{j}\right]=\delta_{k j} a_{k}(\mathbf{X}) \Delta t$ with $\delta_{k j}$ Kronecker's delta. We note that the increments $\Delta Y_{k}$ for $k=1, \ldots, M$ cause the species increment $\Delta X_{i} \equiv \sum_{j=1}^{M} N_{i j} \Delta Y_{j}$. Let's denote $\mathbf{D}[\cdot]$ as the operator that reshapes a vector into a diagonal matrix of appropriate size. We can now approximate the increment $\Delta \mathbf{X}$ with a Gaussian of appropriate mean and variance

$$
\Delta \mathbf{X}=\mathbf{N a}(\mathbf{X}) \Delta t+\mathbf{N}(\mathbf{D}[\mathbf{a}(\mathbf{X})])^{\frac{1}{2}} \Delta \mathbf{W},
$$

with the $M$-dimensional random vector $\Delta \mathbf{W} \sim \mathcal{N}(\mathbf{0}, \mathbf{I} \Delta t)$. Equation (6) corresponds exactly to the Euler-Maruyama discretization [6] of the underlying Ito stochastic differential equation

$$
\mathrm{d} \mathbf{X}=\mathbf{N a}(\mathbf{X}) \mathrm{d} t+\mathbf{N}(\mathbf{D}[\mathbf{a}(\mathbf{X})])^{\frac{1}{2}} \mathrm{~d} \mathbf{W},
$$

with $\mathrm{d} \mathbf{W}$ the increment of the $M$-dimensional Brownian motion. Equation (7) is usually referred to the chemical Langevin equation [5]. The first term on the right-hand-side of (7) corresponds to the deterministic drift term whereas the second term is the stochastic diffusion term with the statedependent diffusion matrix $(\mathbf{D}[\mathbf{a}(\mathbf{X})])^{\frac{1}{2}}$. Clearly in the case of no diffusion we recover the rate equation (5).

The diffusion approximation of the CTMJP yields an approximation of $p(\mathbf{X}, t)$ via the Fokker-Planck equation and a way to generate stochastic sample paths using (7). Although (3) is not solveable in practice for systems of modest size there exists an efficient Monte Carlo sampling scheme [12] to draw sample paths from the exact $p(\mathbf{X}, t)$. Numerous alterations of the scheme to improve simulation speed has been made. See for instance [13] for the important attempt to cope with the problem of stiffness often inherent in bio-molecular reaction systems.

In computer science different efficient representations of this Markov process are available. A one-to-one pendant to the classical reaction representation is that of a stochastic Petri net [14]. Extension thereof that allow to specify reactions only within a local context are rule-based or agent-based models [15]. Those are particularly well suited to cope with highly combinatorial reactions as encountered in signal-transduction [16].

\section{Metabolic Networks ANALYsis}

Metabolism is the sum total of all chemical reactions involved in maintaining the living state of a cell. In general metabolism may be divided into two categories, i.e., catabolism or the break down of molecules to obtain energy and anabolism or the synthesis of all compounds needed by the cell. The key players within metabolic networks are enzymes that catalyze conversion reactions that are otherwise very unlikely to happen. For such enzymatic reactions efficient aggregation of elementary mass-action kinetic laws (2) can be achieved. The most prominent among them is the MichaelisMenten rate law [17]. The copy number of compounds within metabolic networks are usually large and the rate equation approximation (5) is well justified. This high copy number and their relevance for biotechnology make metabolic networks intensively studied quantitatively and genome-scale networks have been mapped out for many organisms [4].

The stoichiometric matrix $\mathbf{N}$ captures the topology of the network and is equivalent to the path-incidence matrix in graph theory. Metabolic networks are usually modeled as nonautonomous systems as they process some substrate metabolite (e.g. glucose) and convert it into some product metabolite (e.g. pyruvate). Thus in accordance with (5) one considers

$$
\frac{\mathrm{d} \hat{\mathbf{x}}}{\mathrm{d} t}=\mathbf{N a}(\hat{\mathbf{x}})+\mathbf{B u}
$$

where the input $\mathbf{u} \in \mathbb{R}^{L}$ are referred to as exchange fluxes that cross the systems boundary. Due to the scarcity of information on kinetic parameters and on concentration levels (or copy numbers $\hat{\mathbf{x}}$ ) a large body of recent work in metabolic network analysis is concerned with the reconstruction of the distribution of fluxes $v_{j} \equiv a_{j}(\hat{\mathbf{x}})$ with $1 \leq j \leq M$ at the steady state $\overline{\mathbf{x}}$ of the network, i.e. $\frac{\mathrm{d} \hat{\mathrm{x}}}{\mathrm{d} t}=\mathbf{0}$. To parallel electrical network analysis one can associate fluxes $v_{j}$ with electrical currents and concentrations (or copy numbers $\hat{\mathbf{x}}$ ) with voltages. The rate law $v_{j}=a_{j}(\hat{\mathbf{x}})$ would thus correspond to a nonlinear $i-v$ characteristic. With (8) we write the steady state condition as

$$
\tilde{\mathbf{N}} \tilde{\mathbf{v}} \equiv\left(\begin{array}{ll}
\mathbf{N} & \mathbf{B}
\end{array}\right)\left(\begin{array}{l}
\overline{\mathbf{v}} \\
\mathbf{u}
\end{array}\right)=\mathbf{0}
$$

with $\overline{\mathbf{v}} \equiv \mathbf{a}(\overline{\mathbf{x}})$ and the implicit definition of the extended stoichiometric matrix $\tilde{\mathbf{N}}$ and extended flux vector $\tilde{\mathbf{v}}$. Thus any vector in the right null-space of $\tilde{\mathbf{N}}$ is an admissible steady state flux vector. The dimension of the null space is $r=\tilde{M}-\operatorname{rank}(\tilde{\mathbf{N}})$ with $\tilde{M} \equiv M+L$. Collecting the $r$ linearly independent non-unique basis vectors $\mathbf{k}_{j}$ of that null 
space into the matrix $\mathbf{K} \equiv\left(\mathbf{k}_{1}, \ldots, \mathbf{k}_{r}\right)$ we have $\tilde{\mathbf{N K}}=$ 0. Assuming that all reactions are irreversible (which can always be done by separating reversible ones into forward and backward reactions) we require that $\tilde{v}_{j} \geq 0$ for $1 \leq j \leq \tilde{M}$. The set of all admissible steady state flux vectors is the convex polyhedral cone [18]

$\mathcal{C}=\left\{\tilde{\mathbf{v}} \in \mathbb{R}^{\tilde{M}} \mid \tilde{\mathbf{v}} \in \operatorname{span}\left(\mathbf{k}_{1}, \ldots, \mathbf{k}_{r}\right) \quad\right.$ with $\left.\quad \tilde{v}_{j} \geq 0 \quad \forall j\right\}$.

In other words the set of admissible $\tilde{\mathbf{v}}$ is the intersection of the null space of $\tilde{\mathbf{N}}$ with the positive orthant. The set $\mathcal{C}$ can further be constrained by incorporating known bounds on maximal fluxes and measured fluxes for particular reactions. Moreover flux balance analysis (FBA) introduces optimality conditions on $\tilde{\mathbf{v}}$ to further constrain $\mathcal{C}$. Criteria such as maximum growth or maximal yield (biomass per uptake flux), or yield of a particular metabolic compound have been used. In general criteria are chosen that result in the linear program

$$
\max _{\tilde{\mathbf{v}} \in \mathcal{C}} \sum_{j=1}^{\tilde{M}} \gamma_{j} \tilde{v}_{j},
$$

with the constant weight vector $\gamma_{k}$ accounting for a particular combination of fluxes, e.g., a combination that corresponds to the biosynthetic requirements for a given biomass composition. To this end quadratic programming gets applied if one uses the criteria of minimal metabolic adjustment [19] which is considered in conjunction with in silico gene deletion experiments. Justifications of those optimization approaches for network reconstruction are based on evolutionary arguments. Good correspondence between the so computed flux distribution and experimental findings have been reported for E. coli [20] and other bacteria. In general this approach is referred to as constraint-based modeling. More can be read off from the stoichiometric matrix. The left null-space of $\tilde{\mathbf{N}}$ describes linear combination of metabolites that are invariants of motion, i.e.

$$
\mathbf{g}^{T} \tilde{\mathbf{N}}=0 \quad \text { and thus } \quad \mathbf{g}^{T} \frac{\mathrm{d} \hat{\mathbf{x}}}{\mathrm{d} t}=\frac{\mathrm{d}}{\mathrm{d} t}\left(\mathbf{g}^{T} \hat{\mathbf{x}}\right)=0 .
$$

To be biological meaningful the elements of $g$ must be restricted to integers $g_{k} \in \mathbb{Z}$. The number of invariants is $\ell=N-\operatorname{rank}(\tilde{\mathbf{N}})$. The rays or edges $\mathbf{f}_{j}$ of the cone $\mathcal{C}$ are of particular importance and unique up to scaling. As $\mathcal{C}$ is convex any admissible flux distribution $\tilde{\mathbf{v}}$ lies in the convex hull of these extreme rays

$$
\tilde{\mathbf{v}}=\sum_{j=1}^{Q} \alpha_{j} \mathbf{f}_{j} \quad \text { with } \quad \alpha_{j} \geq 0 \quad \forall j,
$$

where $Q$ denotes the number of extreme rays. Fluxes $\mathbf{f}_{j} \geq \mathbf{0}$ (elementwise) are also referred to as extreme pathways (EP) or elementary flux modes (EFM) (although they are slightly different [4]). They constitute independent and minimal routes from a particular substrate to a particular product. Minimal in the sense that a deletion of any reaction in the pathway $\mathbf{f}_{j}$ causes a disconnect of this route. Due these features and their uniqueness, EP/EFM facilitate our functional understanding of a metabolic network. The computation of them was shown to be NP-hard and the corresponding analysis can only be done for networks of moderate size. There are several extension of FBA. One of them is structural kinetic modeling [21] that proposes a first-order dynamic extension of FBA to assess the stability of the computed fluxes $\tilde{\mathbf{v}}$.

\section{CONCLUSION}

The paper gives an engineering-centered introduction to the mathematical tools employed in the emerging fields of systems and synthetic biology. We explain the notion of a chemical reaction, its probabilistic description in terms of a continuoustime Markov jump process and its approximation through rate ordinary differential equation. The mathematical basis for the topological analysis of stoichiometric networks is discussed.

\section{ACKNOWLEDGMENT}

H. K. acknowledges the support from the Swiss National Science Foundation, grant no. 200020-117975/1.

\section{REFERENCES}

[1] B. Alberts, A. Johnson, J. Lewis, M. Raff, K. Roberts, and W. P. Molecular biology of the cell, 5th ed. Garland Science, 2008.

[2] J. Tian et al., "Accurate multiplex gene synthesis from programmable DNA microchips," Nature, vol. 432, pp. 1050-1054, 2004.

[3] B. Canton, A. Labno, and D. Endy, "Refinement and standardization of synthetic biological parts and devices," Nature Biotechnology, vol. 26, no. 7, pp. 787-793, 2008.

[4] B. O. Palsson, Systems biology - properties of reconstructed networks. Cambridge University Press, 2007.

[5] D. T. Gillespie, "The chemical Langevin equation," Journal of Chemical Physics, vol. 113, no. 1, pp. 297-306, 2000.

[6] D. J. Wilkinson, Stochastic modelling for systems biology. Chapman \& Hall, 2006.

[7] A. P. Minton, "Macromolecular crowding," Current Biology, vol. 16, no. 8, pp. 269-271, 2006.

[8] J. D. Murray, Mathematical Biology II, 3rd ed. Springer, 2008.

[9] S. S. Andrews and D. Bray, "Stochastic simulation of chemical reactions with spatial resolution and single molecule detail," Physical Biology, vol. 1, pp. 137-151, 2004.

[10] D. T. Gillespie, "A rigorous derivation of the chemical master equation," Physica A, vol. 188, pp. 404-425, 1992.

[11] N. G. Van Kampen, Stochastic processes in physics and chemistry. North-Holland, 2007.

[12] D. T. Gillespie, "Exact stochastic simulation of coupled chemical reactions," Journal of Physical Chemistry, vol. 81, pp. 2340-2361, 1977.

[13] Y. Cao, D. Gillespie, and L. Petzold, "The slow-scale stochastic simulation algorithm," Journal of Chemical Physics, vol. 122, no. 1, p. 014116 , 2005.

[14] J. Fisher and T. Henzinger, "Executable cell biology," Nature Biotechnology, vol. 25, no. 11, pp. 1239-1249, 2007.

[15] W. Hlavacek, J. Faeder, M. Blinov, R. Posner, M. Hucka, and W. Fontana, "Rules for modeling signal-transduction systems," Science STKE, vol. 2006, no. 344, 2006

[16] H. Conzelmann, J. Saez-Rodriguez, T. Sauter, B. Kholodenko, and E. Gilles, "A domain-oriented approach to the reduction of combinatorial complexity in signal transduction networks," BMC Bioinformatics, vol. 7, no. 1, p. 34, 2006.

[17] A. Cornish-Bowden, Fundamentals of enzyme kinetics. Portland Press, 2004.

[18] D. P. Bertsekas, A. Nedic, and A. E. Ozdaglar, Convex analysis and optimization. Athena Scientific, 2003.

[19] D. Segre, D. Vitkup, and G. M. Church, "Analysis of optimality in natural and perturbed metabolic networks," Proceeding of the National Academy of Sciences of the USA, vol. 99, no. 23, pp. 15112-15117, 2002.

[20] J. S. Edwards, R. U. Ibarra, and B. O. Palsson, "In silico prediction of Escherichia coli metabolic capabilities are consistent with experimental data," Nature Biotechnology, vol. 19, no. 0, pp. 125-130, 2001.

[21] R. Steuer, T. Gross, and B. Balsius, "Structural kinetic modeling of metabolic networks," Proceeding of the National Academy of Sciences of the USA, vol. 103, no. 32, pp. 11868-11873, 2006. 